\title{
A five-gene based risk score with high prognostic value in colorectal cancer
}

\author{
YIDA PAN $^{1 *}$, HONGYANG ZHANG $^{1 *}$, MINGMING ZHANG $^{2}$, JIE ZHU $^{1}$, \\ JIANGHONG YU ${ }^{1,3}$, BANGTING WANG ${ }^{1}$, JIGANG QIU $^{4}$ and JUN ZHANG ${ }^{1}$
}

${ }^{1}$ Department of Digestive Diseases, Huashan Hospital, Fudan University, Shanghai 200040; ${ }^{2}$ Department of Gastroenterology, Nanjing Drum Tower Hospital, Nanjing University, Nanjing 210008; ${ }^{3}$ Institutes of Biomedical Sciences, Fudan University, Shanghai 200032; ${ }^{4}$ Department of General Surgery, Huadong Hospital, Fudan University, Shanghai 200040, P.R. China

Received March 4, 2017; Accepted August 31, 2017

DOI: $10.3892 / 01.2017 .7097$

\begin{abstract}
Colorectal cancer (CRC) is one of the most frequently occurring malignancies worldwide. The outcomes of patients with similar clinical symptoms or at similar pathological stages remain unpredictable. This inherent clinical diversity is most likely due to the genetic heterogeneity. The present study aimed to create a predicting tool to evaluate patient survival based on genetic profile. Firstly, three Gene Expression Omnibus (GEO) datasets (GSE9348, GSE44076 and GSE44861) were utilized to identify and validate differentially expressed genes (DEGs) in CRC. The GSE14333 dataset containing survival information was then introduced in order to screen and verify prognosis-associated genes. Of the 66 DEGs, the present study screened out 46 biomarkers closely associated to patient overall survival. By Gene Ontology and Kyoto Encyclopedia of Genes and Genomes pathway analysis, it was demonstrated that these genes participated in multiple biological processes which were highly associated with cancer proliferation, drug-resistance and metastasis, thus further affecting patient survival. The five most important genes, MET proto-oncogene, receptor tyrosine kinase, carboxypeptidase $\mathrm{M}$, serine hydroxymethyltransferase 2 , guanylate cyclase activator $2 \mathrm{~B}$ and sodium voltage-gated channel a subunit 9 were selected by a random survival forests algorithm, and were further made up to a linear risk score formula by multivariable cox regression. Finally, the present
\end{abstract}

Correspondence to: Dr Jun Zhang, Department of Digestive Diseases, Huashan Hospital, Fudan University, 12 Middle Wulumuqi Road, Shanghai 200040, P.R. China

E-mail: archsteed@gmail.com

Dr Jigang Qiu, Department of General Surgery, Huadong Hospital, Fudan University, 221 West Yan'an Road, Shanghai 200040, P.R. China

E-mail: qiujigang@126.com

${ }^{*}$ Contribute equally

Key words: colorectal cancer, microarray, DEG, overall survival, risk score study tested and verified this risk score within three independent GEO datasets (GSE14333, GSE17536 and GSE29621), and observed that patients with a high risk score had a lower overall survival $(\mathrm{P}<0.05)$. Furthermore, this risk score was the most significant compared with other predicting factors including age and American Joint Committee on Cancer stage, in the model, and was able to predict patient survival independently and directly. The findings suggest that this survival associated DEGs-based risk score is a powerful and accurate prognostic tool and is promisingly implemented in a clinical setting.

\section{Introduction}

Colorectal cancer (CRC) is currently one of the most commonly diagnosed cancers worldwide, with an estimated 1.4 million cases and 693,900 deaths occurring in 2012 (1). It is much more prevalent in Europe and Northern America than the developing countries, which however is also rising in the last decade (2). Though many advances have been achieved in the clinical management of $\mathrm{CRC}$, the 5-year survival is usually only approximately 55\% (3). Surgical resection remains the primary means of curative treatment. However, a proportion of patients will develop local recurrences and metastases thus having a poor prognosis after resection. Moreover, the outcomes of patients with similar clinical or pathologic stage remain unpredictable, especially when they are treated similarly (4). This inherent clinical diversity is most likely due to the genetic heterogeneity of each patient (5). Therefore, identifying the diversity in the genetic profile of colorectal carcinoma that governs the prognosis as well as accurate risk evaluation based on genetic screening would lead to new and more effective clinical strategies in decision making.

Microarray technology allows comprehensive analysis of gene expression profiles in different diseases, which has been demonstrated in a variety of hematological tumors and solid tumors including lung (6), liver (7), pancreas (8), and breast (9). Biomarkers discovered by microarrays have a great potential in the prediction of clinical outcomes and survival as well as classification in different sub-types (10-12). However, several reported survival-related biomarkers in CRC are not well performed when their ability was assessed in independent datasets (13-15). Their clinical implement may also limited due to lack of reproducibility 
and/or standardization. This may be related to un-optimized parameters, different technique platforms, and small volume of samples. So an integrated strategy to combine several specific biomarkers together, which are verified by multiple data source, may be feasible in predicting CRC risk and prognosis.

In the present study, we identified and verified 66 differentially expressed genes (DEGs) between CRC and normal tissue by bioinformatics analysis with multiple classifiers. Among them, we classified 46 biomarkers which were closely related to patient survival. We looked into the function of these genes via GO and KEGG pathway analysis. Finally, through random survival forests algorithm, we ranked these gene by importance and built a 5-genes-based linear risk score with multivariable cox regression model. Our findings suggest that this risk score is a powerful and arcuate prognostic tool and is promisingly implemented in the clinical setting.

\section{Materials and methods}

CRC datasets. The training and validation datasets were achieved from the Gene Expression Omnibus (GEO, https://www.ncbi. nlm.nih.gov/geo/). GSE9348 (70 CRC and 12 normal, platform GPL570 [HG-U133_Plus_2] Affymetrix Human Genome U133 Plus 2.0 Array) was used as training set for DEGs to distinguish cancerous and non-cancerous samples, GSE44076 (98 pairs of CRC and adjacent normal tissues, platform GPL13667 [HG-U219] Affymetrix Human Genome U219 Array) and GSE44861 (56 tumors and 55 adjacent normal tissues, by GPL3921 [HT_ HG-U133A] Affymetrix HT Human Genome U133A Array) for validation. Three datasets with survival information generated by GPL570 [HG-U133_Plus_2] Affymetrix Human Genome U133 Plus 2.0 Array were introduced for calculating risk score formula. GSE14333 ( $n=226$ ) was set for training set, and GSE17536 ( $n=177)$ as well as GSE29621 $(n=65)$ for validation.

Data preprocessing. All microarray data preprocessing were processed in $\mathrm{R}$ software version 3.1.0 using packages from Bioconductor. Raw microarray data (CEL files) of tumors and normal samples were pre-processed with the RMA algorithm using the affy package (16). Gene expression values were arranged after background adjustment, quantile normalization and summarizing probe values into one expression measure. If multiple probe sets mapping to a same gene, the averages of the probe values were taken as the expression values (17). Annotations for the probe arrays were downloaded from the GEO database.

Functional enrichment analysis. The GO and pathway functional enrichment analysis was operated by the online software GENECODIS3 to facilitate the interpretation of biological roles of survival related-DEGs (http://genecodis.cnb.csic.es) (18). The GO functions of the survival related-DEGs were categorized by biological process, molecular functions, and cellular components. Pathway enrichment analysis was based on the Kyoto Encyclopedia of Genes and Genomes (KEGG) database. P-values have been obtained through Hypergeometric analysis corrected by FDR method. Terms with $\mathrm{P}<0.05$ were considered as significantly enriched.

Statistical analysis. SPSS software (version 20.0; IBM SPSS, Armonk, NY, USA) were applied for statistical analysis.
Survival analysis was performed by Kaplan-Meier method and Mantel-Cox log-rank test was used to evaluate the statistical significance of the differences. Pearson's Chi-Square test was used to investigate the difference in live and dead status of patients with different risk score. Differences were considered as statistically significant when $\mathrm{P}<0.05$.

\section{Results}

Identification of DEGs between cancerous and non-cancerous tissues. GSE9348 was used as the training set to identify the DEGs between cancerous and non-cancerous tissues. This dataset included tumors from 70 patients and biopsies from 12 healthy controls. We employed different classifiers, namely Compound Covariate (CC), Diagonal Linear Discriminant Analysis (DLDA), Bayesian CCP (BCCP), Nearest Neighbor (NN), Nearest Centroid (NC) and Support Vector Machines (SVM), to identify specific gene markers. Leave-one-out cross validation was introduced to make the result stable and accurate. After processing, we got 66 DEGs, with high accuracy (classifier error rate $<0.1$ ) (data not shown). The distribution of the 66 genes in tumor and non-tumor tissue was clearly demarcated in the GSE9348 dataset (Fig. 1A). To further confirm the DEGs in cancerous and non-cancerous tissue, the human protein atlas immunohistochemistry database (www.proteinatlas.org) was utilized to visualize the expression. We found that downregulated DEGs like SCN9A, UGP2 and CWH43 were less stained even negative in CRC tissues (Fig. 1B), while upregulated DEGs as MET, MYC and SHMT2 were high stained in tumor parts (Fig. 1C).

As classifier CC, DLDA and SVM were linear classifiers, a linear discriminant with weight values could determine the cancerous status of samples. If one gene's weight value in a sample within a certain linear classifier was $\omega_{i}$, and its expression value $x_{i}$, then $\sum_{i} \omega_{i} x_{i>}$ threshold was defined as cancerous. The threshold of classifier CC, DLDA and SVM were calculated as $-43.835,-234.08$ and 0.409 , respectively. The ROC curves of the three linear classifiers confirmed its high effectiveness $(A U C=1)$ (Fig. 1D). It should be noted that these ROC curves were derived from the training set GSE9348, in which the $\sum_{i} \omega_{i} x_{i}$ discriminant of the three linear classifiers was set to compare with a calculated threshold adapting to GSE9348, so the sensibility and specificity was very high (Table I upper).

Validation of DEGs in independent CRC datasets. To avoid over-fitting and ensure marker stability, two independent CRC datasets, GSE44076 (98 pairs of CRC and adjacent tissues) and GSE44861 (56 tumors and 55 adjacent tissues) were introduced for verification. The classifiers utilized in GSE9348 worked well in these datasets (Table II), and the sensibility and specificity of $\Sigma_{i} \omega_{i} x_{i}$ discriminant in classification of cancerous samples were also tested and confirmed (Table I middle and lower). Gene expressions of the 66 DEGs derived from GSE9348 performed a similar style in GSE44076 and GSE44861 (data not shown). The reliability of the three linear classifiers (CC, DLDA and SVM) was guaranteed when they applied to GSE44076 and GSE44861. The AUCs of classifier CC, DLDA and SVM in GSE44076 were 0.9994, 0.9996 and 0.9994 (Fig. 2A), while in GSE44861 the AUC values were 0.9253, 0.9292 and 0.9318, respectively (Fig. 2B). 
Table I. Survival related DEGs by univariable cox proportional hazards regression analysis.

\begin{tabular}{|c|c|c|c|c|c|}
\hline Gene & P-value & HR & Gene & P-value & HR \\
\hline LOC339166 & $<1 \mathrm{e}-07$ & 7.748 & MYC & 7E-07 & 0.581 \\
\hline SCN9A & $<1 \mathrm{e}-07$ & 0.154 & SQRDL & 7E-07 & 0.513 \\
\hline LGI1 & $<1 \mathrm{e}-07$ & 0.115 & SHMT2 & 0.000001 & 0.509 \\
\hline P2RY1 & $<1 \mathrm{e}-07$ & 3.592 & PDE6A & $2.1 \mathrm{E}-06$ & 2.229 \\
\hline PRPF4 & $<1 \mathrm{e}-07$ & 0.245 & UGDH & 2.3E-06 & 1.792 \\
\hline GUCA2B & $<1 \mathrm{e}-07$ & 1.688 & PTPRH & $2.5 \mathrm{E}-06$ & 1.733 \\
\hline ENOX2 & $<1 \mathrm{e}-07$ & 0.193 & PPP2R3A & $8.4 \mathrm{E}-06$ & 2.19 \\
\hline NPY & $<1 \mathrm{e}-07$ & 4.787 & HSPH1 & $2.62 \mathrm{E}-05$ & 1.61 \\
\hline SCGN & $<1 \mathrm{e}-07$ & 2.266 & NR5A2 & $3.16 \mathrm{E}-05$ & 0.585 \\
\hline TMEM9B & $<1 \mathrm{e}-07$ & 3.445 & TRIP13 & $3.21 \mathrm{E}-05$ & 0.631 \\
\hline RNASEH2A & $<1 \mathrm{e}-07$ & 0.438 & CPM & $6.06 \mathrm{E}-05$ & 0.498 \\
\hline HSD11B2 & $<1 \mathrm{e}-07$ & 0.647 & DUSP14 & 0.000183 & 0.54 \\
\hline DENND2A & $<1 \mathrm{e}-07$ & 0.299 & RCL1 & 0.000274 & 0.415 \\
\hline ASPA & $<1 \mathrm{e}-07$ & 3.507 & ETV4 & 0.000396 & 0.672 \\
\hline CA7 & $<1 \mathrm{e}-07$ & 2.626 & SEMA6D & 0.000472 & 1.9 \\
\hline LPHN3 & $<1 \mathrm{e}-07$ & 0.247 & HOMER 1 & 0.000475 & 0.666 \\
\hline ABCG2 & $<1 \mathrm{e}-07$ & 1.497 & CCND1 & 0.000522 & 1.584 \\
\hline GALNT6 & $<1 \mathrm{e}-07$ & 0.588 & METTL7A & 0.000543 & 2.012 \\
\hline PTGDR & $<1 \mathrm{e}-07$ & 0.336 & MET & 0.000577 & 1.528 \\
\hline $\mathrm{TST}$ & $<1 \mathrm{e}-07$ & 0.497 & CWH43 & 0.0006 & 0.699 \\
\hline SMPDL3A & 1E-07 & 0.428 & DHRS11 & 0.000607 & 0.748 \\
\hline HSD17B11 & $1 \mathrm{E}-07$ & 2.087 & UGP2 & 0.000701 & 1.977 \\
\hline ETFDH & $3 \mathrm{E}-07$ & 0.549 & SLC22A18AS & 0.000812 & 0.558 \\
\hline
\end{tabular}

HR, hazard ratio.

Survival analysis of DEGs in CRC and their function annotation. The 66 biomarkers were significant differential genes in CRC, however, whether the expression of these genes were correlated with patient survival was unclear. We used GSE14333 which contained 226 samples with survival information among total 290 patients as the training set for survival analysis. By univariable cox proportional hazards regression analysis and random permutation test, we obtained 46 genes correlated with patient survival $(\mathrm{P}<0.001)$ (Table III).

To elucidate the function of these survival related DEGs, we conducted GO and KEGG pathway analysis and revealed that many genes play an important role in 'response to drug', 'metabolic process', 'cell proliferation', and 'oxidoreductase activity', which were highly correlated to drug resistance, altered cancer metabolism, ROS level and proliferation, and many genes also participated in multiple cancer pathways, such as MYC and CCND1 (Table IV).

Construction of risk score formula. In order to select the most weighted genes, we utilized random survival forests algorithm (Ntree $=1,000$, default parameters of Hemant Ishwaran algorithm) (Fig. 3A), and set the 46 survival related genes as variables in this model. We ranked these 46 genes by their importance after the processing of random survival forests algorithm via R software (Fig. 3B). Five genes, namely MET, CPM, SHMT2, GUCA2B and SCN9A were selected as the most important candidates (relative importance $>0.5$ ). Relative importance means the relative value of a certain gene normalized to the gene MET, which was the most important gene in our random survival forests model (Fig. 3B, and detailed normalized data not shown). To investigate whether the 5 candidates could provide an accurate prediction of survival in CRC patients, the expression data of these genes were fit into a multivariable cox regression model as covariates of the training dataset. We obtained each gene's regression coefficient and then built a risk score formula for each individual as follows:

Risk score $=-0.370^{*}$ (expression value of CPM $)-0.122^{*}$ (expression value of GUCA2B) $+0.332^{*}$ (expression value of MET) $+0.088^{*}$ (expression value of SCN9A) $+0.827^{*}$ (expression value of SHMT2).

Cutting off by the median of the risk score, we defined risk score $<$ median as low-risk group, and risk score $>$ median as high-risk group. To assess the reliability of the risk-score formula in predicting patients survival, we ranked all the patients in the training set GSE14333, and divided them into either high-risk group $(n=116)$ or low-risk group $(n=113$; Fig. 4). Patients in the low-risk group had a markedly longer overall survival than those in the high-risk group $(\mathrm{P}=0.001$, by Mantel-Cox log rank) (Fig. 4A). The distribution of the follow-up months of a certain risk score and the live/dead status were shown in Fig. 4B. However, the P-value by Pearson Chi-Square test was 0.109, suggesting no significant difference between the live and dead status of patients with different risk score, indicating that our work was more valuable in predicting 
Table II. GO analysis and KEGG pathway analysis of 46 survival related-DEGs (partial data).

\begin{tabular}{|c|c|c|c|}
\hline Genes & Hyp & Hyp $^{\mathrm{a}}$ & Annotations \\
\hline \multicolumn{4}{|c|}{ Biological process } \\
\hline 5 & 4.7E-05 & 0.00408 & GO:0042493: Response to drug (BP) \\
\hline 4 & 0.00079 & 0.02956 & GO:0008152: Metabolic process (BP) \\
\hline 3 & 0.00804 & 0.03142 & GO:0008283: Cell proliferation (BP) \\
\hline 3 & 0.00769 & 0.03249 & GO:0007411: Axon guidance (BP) \\
\hline 3 & 0.01192 & 0.0359 & GO:0008284: Positive regulation of cell proliferation (BP) \\
\hline 3 & 0.02362 & 0.04835 & $\begin{array}{l}\text { GO:0045893: Positive regulation of transcription, } \\
\text { DNA-dependent }(\mathrm{BP})\end{array}$ \\
\hline \multicolumn{4}{|c|}{ Molecular function } \\
\hline 13 & 0.00391 & 0.02429 & GO:0005515: Protein binding (MF) \\
\hline 7 & $1.6 \mathrm{E}-06$ & 0.00019 & GO:0016491: Oxidoreductase activity (MF) \\
\hline 7 & 0.01988 & 0.04888 & GO:0000166: Nucleotide binding (MF) \\
\hline 6 & 0.01607 & 0.0431 & GO:0004872: Receptor activity (MF) \\
\hline 5 & 0.00871 & 0.03213 & GO:0016787: Hydrolase activity (MF) \\
\hline 4 & 0.00865 & 0.03294 & GO:0016740: Transferase activity (MF) \\
\hline 4 & 0.01535 & 0.04312 & GO:0004930: G-protein coupled receptor activity (MF) \\
\hline \multicolumn{4}{|c|}{ Cellular component } \\
\hline 15 & 0.00234 & 0.03334 & GO:0005737: Cytoplasm (CC) \\
\hline 13 & 0.00169 & 0.03219 & GO:0016020: Membrane (CC) \\
\hline 11 & 0.00562 & 0.03205 & GO:0005886: Plasma membrane (CC) \\
\hline 9 & 0.00075 & 0.02125 & GO:0005576: Extracellular region (CC) \\
\hline 7 & 0.0028 & 0.02656 & GO:0005730: Nucleolus (CC) \\
\hline 6 & 0.00948 & 0.04156 & GO:0005739: Mitochondrion (CC) \\
\hline 5 & 0.00357 & 0.02911 & GO:0005615: Extracellular space (CC) \\
\hline 4 & 0.00072 & 0.04092 & GO:0005743: Mitochondrial inner membrane (CC) \\
\hline 3 & 0.00236 & 0.02696 & GO:0005759: Mitochondrial matrix (CC) \\
\hline \multicolumn{4}{|c|}{ KEGG pathway } \\
\hline 3 & 0.0089 & 0.0411 & (KEGG) 05200: Pathways in cancer \\
\hline 2 & 0.00215 & 0.02152 & (KEGG) 05213: Endometrial cancer \\
\hline 2 & 0.00258 & 0.02211 & (KEGG) 05221: Acute myeloid leukemia \\
\hline 2 & 0.00304 & 0.02282 & (KEGG) 05210: Colorectal cancer \\
\hline 2 & 0.00077 & 0.02304 & (KEGG) 00040: Pentose and glucuronate interconversions \\
\hline 2 & 0.00207 & 0.02485 & (KEGG) 00500: Starch and sucrose metabolism \\
\hline 2 & 0.00419 & 0.02514 & (KEGG) 05220: Chronic myeloid leukemia \\
\hline 2 & 0.00386 & 0.02574 & (KEGG) 05218: Melanoma \\
\hline 2 & 0.00184 & 0.02755 & (KEGG) 00520: Amino sugar and nucleotide sugar metabolism \\
\hline 2 & 0.00141 & 0.02818 & (KEGG) 05219: Bladder cancer \\
\hline 2 & 0.00551 & 0.03004 & (KEGG) 05222: Small cell lung cancer \\
\hline 2 & 0.00063 & 0.03755 & (KEGG) 05216: Thyroid cancer \\
\hline 2 & 0.01148 & 0.04919 & (KEGG) 04110: Cell cycle \\
\hline 2 & 0.01238 & 0.04953 & (KEGG) 04360: Axon guidance \\
\hline
\end{tabular}

Partial data, genes involved $\geq 3$ (GO analysis) or gene involved $\geq 2$ (KEGG pathway analysis). Genes involved in all KEGG pathway above were MYC and CCND1. ${ }^{a}$ corrected Hyp. Hyp, hypergeometric P-value; BP, biological processes; MF, molecular function; CC, cellular component.

patient overall survival (Fig. 4A), not the final live/dead status. Moreover, the distribution of risk score in lower expression of SCN9A, CPM and GUCA2B as well as higher expression of MET and SHMT2 showed relative homogeneity and stability from patient to patient with high risk score (Fig. 4E upper).
In addition, we performed multivariable and univariate cox regression analysis to elucidate the relationship between risk score and other factors like sex, age of diagnosis and Dukes stage. It was shown that risk score was the most significant among other factors $[\mathrm{P}=0.005$ (multivarible) and $\mathrm{P}=0.001$ 
A

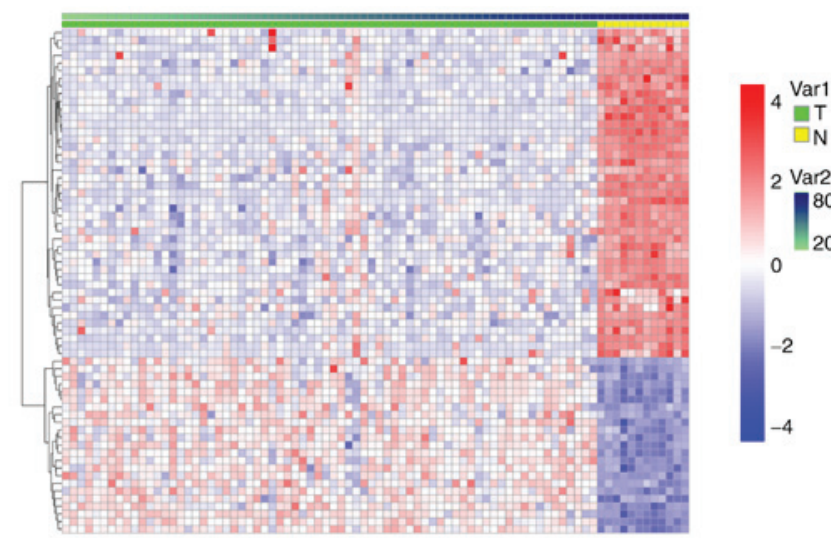

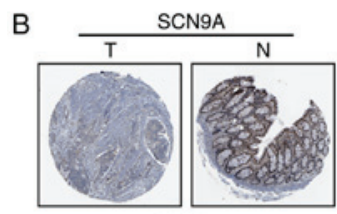
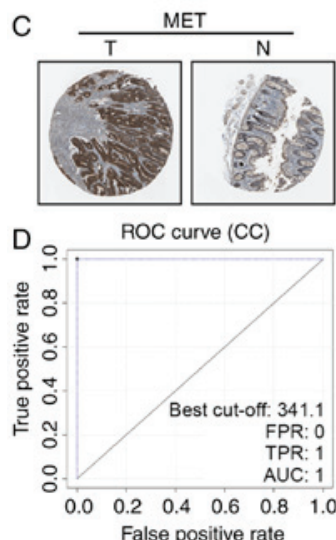

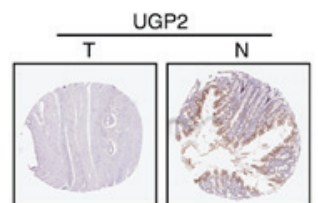

MYC

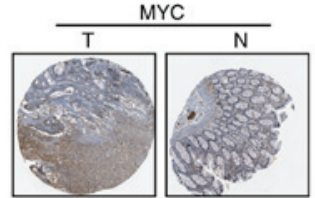

ROC curve (DLDA)

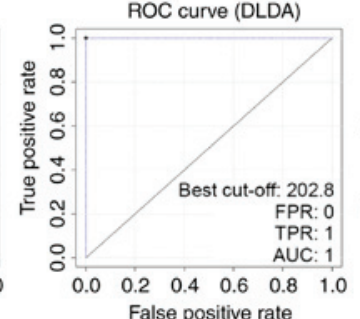

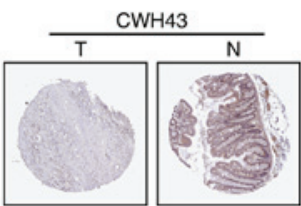
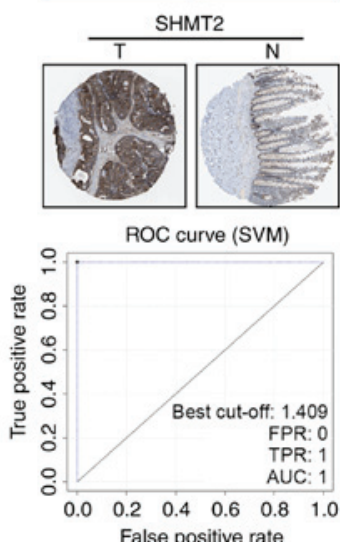

Figure 1. DEGs in colorectal cancer. (A) Heatmap of 66 DEGs expression in cancer and non-cancerous tissue of GSE9348. More detailed information could be achieved by contacting the corresponding author. (B) Immunohistochemistry (IHC) pictures of SCN9A, UGP2 and CWH43 as downregulated DEGs were archieved from the Human Protein Atlas database (HPA). (C) IHC results of MET, MYC and SHMT2 as upregulated DEGs from HPA. (D) ROC curves of three linear classifier CC, DLDA and SVM in training set GSE9348. FPR, false positive rate; TPR, true positive rate; AUC, area under curve; DEGs, Differentially expressed genes; $\mathrm{T}$, tumor; $\mathrm{N}$, normal.
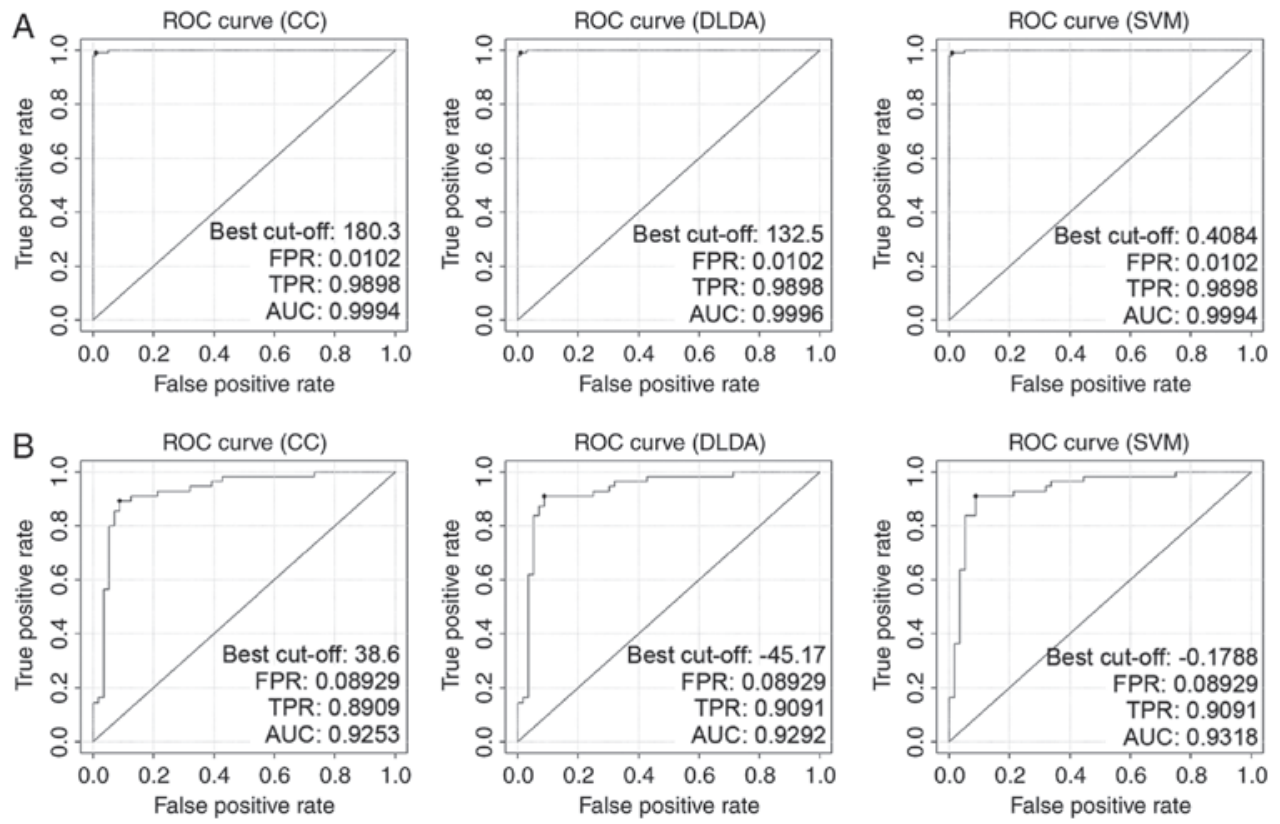

Figure 2. ROC curves of linear classifier CC, DLDA and SVM of validation sets. ROC curves of linear classifier CC, DLDA and SVM in validation sets GSE44076 (A) and GSE44861 (B). FPR, false positive rate; TPR, true positive rate; AUC, area under curve. 
Table III. Multivariable and univariable model tests of risk score and other factors.

\section{A, GSE14333}

\begin{tabular}{|c|c|c|c|c|c|c|c|c|}
\hline \multirow{3}{*}{$\begin{array}{l}\text { Variables } \\
\text { Risk score }\end{array}$} & \multicolumn{4}{|c|}{ Multivariable model } & \multicolumn{4}{|c|}{ Univariable model } \\
\hline & \multirow{2}{*}{$\begin{array}{c}\text { HR } \\
2.346\end{array}$} & \multicolumn{2}{|c|}{$95 \% \mathrm{CI}$ of $\mathrm{HR}$} & \multirow{2}{*}{$\begin{array}{c}\text { P-value } \\
0.005\end{array}$} & \multirow{2}{*}{$\begin{array}{c}\text { HR } \\
2.718\end{array}$} & \multicolumn{2}{|c|}{$95 \% \mathrm{CI}$ of $\mathrm{HR}$} & \multirow{2}{*}{$\begin{array}{l}\text { P-value } \\
0.001\end{array}$} \\
\hline & & 1.298 & 4.241 & & & 1.523 & 4.851 & \\
\hline Location & 0.965 & 0.814 & 1.144 & 0.683 & 0.892 & 0.76 & 1.047 & 0.163 \\
\hline Dukes stage & 1.18 & 0.926 & 1.503 & 0.18 & 1.044 & 0.86 & 1.266 & 0.666 \\
\hline Age of diagnosis & 1.008 & 0.994 & 1.023 & 0.257 & 1.105 & 1.002 & 1.028 & 0.02 \\
\hline Sex & 0.926 & 0.683 & 1.255 & 0.62 & 0.877 & 0.651 & 1.182 & 0.39 \\
\hline Adj XRT & 0.463 & 0.218 & 0.984 & 0.045 & 0.433 & 0.212 & 0.884 & 0.021 \\
\hline Adj CTX & 0.867 & 0.568 & 1.325 & 0.51 & 0.847 & 0.618 & 1.16 & 0.3 \\
\hline
\end{tabular}

\section{B, GSE17536}

\begin{tabular}{|c|c|c|c|c|c|c|c|c|}
\hline \multirow{3}{*}{$\begin{array}{l}\text { Variables } \\
\text { Risk score }\end{array}$} & \multicolumn{4}{|c|}{ Multivariable model } & \multicolumn{4}{|c|}{ Univariable model } \\
\hline & \multirow{2}{*}{$\frac{\mathrm{HR}}{2.745}$} & \multicolumn{2}{|c|}{$95 \% \mathrm{CI}$ of $\mathrm{HR}$} & \multirow{2}{*}{$\begin{array}{c}\text { P-value } \\
0.016\end{array}$} & \multirow{2}{*}{$\frac{\mathrm{HR}}{3.283}$} & \multicolumn{2}{|c|}{$95 \% \mathrm{CI}$ of $\mathrm{HR}$} & \multirow{2}{*}{$\begin{array}{c}\text { P-value } \\
0.003\end{array}$} \\
\hline & & 1.204 & 6.262 & & & 1.489 & 7.236 & \\
\hline Age & 1.015 & 0.999 & 1.031 & 0.061 & 1.018 & 1.003 & 1.034 & 0.016 \\
\hline Sex & 1.084 & 0.747 & 1.572 & 0.672 & 0.953 & 0.666 & 1.362 & 0.79 \\
\hline Ethnicity & 0.967 & 0.728 & 1.284 & 0.817 & 0.915 & 0.685 & 1.221 & 0.545 \\
\hline AJCC stage & 1.107 & 0.892 & 1.373 & 0.357 & 1.051 & 0.861 & 1.284 & 0.625 \\
\hline Grade & 1.254 & 0.828 & 1.898 & 0.285 & 1.375 & 0.924 & 2.045 & 0.116 \\
\hline
\end{tabular}

\section{C, GSE29621}

Multivariable model

Univariable model

\begin{tabular}{|c|c|c|c|c|c|c|c|c|}
\hline \multirow{3}{*}{$\begin{array}{l}\text { Variables } \\
\text { Risk score }\end{array}$} & & & & & \\
\hline & HR & \multicolumn{2}{|c|}{$95 \% \mathrm{CI}$ of $\mathrm{HR}$} & \multirow{2}{*}{$\begin{array}{c}\text { P-value } \\
0.019\end{array}$} & \multirow{2}{*}{$\frac{\mathrm{HR}}{2.526}$} & \multicolumn{2}{|c|}{$95 \% \mathrm{CI}$ of $\mathrm{HR}$} & \multirow{2}{*}{$\frac{\text { P-value }}{2.73 \mathrm{E}-05}$} \\
\hline & 9.03 & 1.425 & 57.223 & & & 0.481 & 13.269 & \\
\hline Sex & 1.243 & 0.513 & 3.014 & 0.63 & 1.508 & 0.649 & 3.505 & 0.34 \\
\hline T stage & 0.449 & 0.091 & 2.209 & 0.325 & 1.048 & 0.438 & 2.509 & 0.915 \\
\hline $\mathrm{N}$ stage & 1.583 & 0.604 & 4.143 & 0.35 & 2.688 & 1.526 & 4.734 & 0.001 \\
\hline M stage & 2.065 & 0.368 & 11.592 & 0.41 & 4.934 & 2.188 & 11.124 & $1.19 \mathrm{E}-04$ \\
\hline Histology grade & 0.849 & 0.325 & 2.219 & 0.738 & 0.665 & 0.284 & 1.558 & 0.348 \\
\hline AJCC stage & 1.965 & 0.518 & 7.45 & 0.321 & 2.708 & 1.615 & 4.542 & $1.59 \mathrm{E}-04$ \\
\hline
\end{tabular}

HR, hazard ratio; Adj XRT, adjuvant radiation therapy; Adj CTX, adjuvant chemotherapy.

(univariable)], while age $(\mathrm{P}=0.016)$ and adjuvant radiation therapy $(\mathrm{P}=0.021)$ were univariable factors to prognosis as reported $(19,20)$ (Table $\mathrm{V}$ upper). These data suggested that the risk score could predict patient survival directly and independently.

Validation of risk score in predicting survival within independent CRC datasets. To further evaluate the clinical value of this risk score, we used 2 independent CRC datasets GSE17536 $(n=177)$ and GSE29621 $(n=65)$ with survival information. We utilized the threshold in GSE14333 to classify high-risk and low-risk groups. Both datasets showed that high risk score patients had lower overall survival $(\mathrm{P}=0.001$, GSE17536; $\mathrm{P}=0.038$, GSE29621) (Fig. 4C and D). The 5 biomarkers of risk score (MET, CPM, SHMT2, GUCA2B and SCN9A) perform a similar stability in GSE17536 and GSE29621 as in GSE14333 (Fig. 4E middle and lower). In addition, by multivariable and univariate cox regression analysis, we confirmed that this risk score was the most significant in GSE17536 [P=0.016 (multivarible) and $\mathrm{P}=0.003$ (univariable)], while $\mathrm{P}$-value of other factors $>0.05$ except age, which was a univariable significant only $(\mathrm{P}=0.016)$ (Table V middle). In GSE29621, risk score 
Table IV. GO analysis and KEGG pathway analysis of 46 survival related-DEGs (partial data).

\begin{tabular}{|c|c|c|c|}
\hline Genes & Hyp & Hyp $^{\mathrm{a}}$ & Annotations \\
\hline \multicolumn{4}{|c|}{ Biological process } \\
\hline 5 & 4.7E-05 & 0.00408 & GO:0042493: Response to drug (BP) \\
\hline 4 & 0.00079 & 0.02956 & GO:0008152: Metabolic process (BP) \\
\hline 3 & 0.00804 & 0.03142 & GO:0008283: Cell proliferation (BP) \\
\hline 3 & 0.00769 & 0.03249 & GO:0007411: Axon guidance (BP) \\
\hline 3 & 0.01192 & 0.0359 & GO:0008284: Positive regulation of cell proliferation (BP) \\
\hline 3 & 0.02362 & 0.04835 & GO:0045893: Positive regulation of transcription, DNA-dependent (BP) \\
\hline \multicolumn{4}{|c|}{ Molecular function } \\
\hline 13 & 0.00391 & 0.02429 & GO:0005515: Protein binding (MF) \\
\hline 7 & $1.6 \mathrm{E}-06$ & 0.00019 & GO:0016491: Oxidoreductase activity (MF) \\
\hline 7 & 0.01988 & 0.04888 & GO:0000166: Nucleotide binding (MF) \\
\hline 6 & 0.01607 & 0.0431 & GO:0004872: Receptor activity (MF) \\
\hline 5 & 0.00871 & 0.03213 & GO:0016787: Hydrolase activity (MF) \\
\hline 4 & 0.00865 & 0.03294 & GO:0016740: Transferase activity (MF) \\
\hline 4 & 0.01535 & 0.04312 & GO:0004930: G-protein coupled receptor activity (MF) \\
\hline \multicolumn{4}{|c|}{ Cellular component } \\
\hline 15 & 0.00234 & 0.03334 & GO:0005737: Cytoplasm (CC) \\
\hline 13 & 0.00169 & 0.03219 & GO:0016020: Membrane (CC) \\
\hline 11 & 0.00562 & 0.03205 & GO:0005886: Plasma membrane (CC) \\
\hline 9 & 0.00075 & 0.02125 & GO:0005576: Extracellular region (CC) \\
\hline 7 & 0.0028 & 0.02656 & GO:0005730: Nucleolus (CC) \\
\hline 6 & 0.00948 & 0.04156 & GO:0005739: Mitochondrion (CC) \\
\hline 5 & 0.00357 & 0.02911 & GO:0005615: Extracellular space (CC) \\
\hline 4 & 0.00072 & 0.04092 & GO:0005743: Mitochondrial inner membrane (CC) \\
\hline 3 & 0.00236 & 0.02696 & GO:0005759: Mitochondrial matrix (CC) \\
\hline \multicolumn{4}{|c|}{ KEGG pathway } \\
\hline 3 & 0.0089 & 0.0411 & (KEGG) 05200: Pathways in cancer \\
\hline 2 & 0.00215 & 0.02152 & (KEGG) 05213: Endometrial cancer \\
\hline 2 & 0.00258 & 0.02211 & (KEGG) 05221: Acute myeloid leukemia \\
\hline 2 & 0.00304 & 0.02282 & (KEGG) 05210: Colorectal cancer \\
\hline 2 & 0.00077 & 0.02304 & (KEGG) 00040: Pentose and glucuronate interconversions \\
\hline 2 & 0.00207 & 0.02485 & (KEGG) 00500: Starch and sucrose metabolism \\
\hline 2 & 0.00419 & 0.02514 & (KEGG) 05220: Chronic myeloid leukemia \\
\hline 2 & 0.00386 & 0.02574 & (KEGG) 05218: Melanoma \\
\hline 2 & 0.00184 & 0.02755 & (KEGG) 00520: Amino sugar and nucleotide sugar metabolism \\
\hline 2 & 0.00141 & 0.02818 & (KEGG) 05219: Bladder cancer \\
\hline 2 & 0.00551 & 0.03004 & (KEGG) 05222: Small cell lung cancer \\
\hline 2 & 0.00063 & 0.03755 & (KEGG) 05216: Thyroid cancer \\
\hline 2 & 0.01148 & 0.04919 & (KEGG) 04110: Cell cycle \\
\hline 2 & 0.01238 & 0.04953 & (KEGG) 04360: Axon guidance \\
\hline
\end{tabular}

Partial data, genes involved $\geq 3$ (GO analysis) or gene involved $\geq 2$ (KEGG pathway analysis). Genes involved in all KEGG pathway above were MYC and CCND1. a, corrected hypergeometric P-value; Hyp, Hypergeometric P-value; KEGG, Kyoto Encyclopedia of Genes and Genomes; BP, biological processes; MF, molecular function; CC, cellular component.

was also the most significant $(\mathrm{P}=0.019$ (multivarible) and $\mathrm{P}=2.73 \mathrm{E}-05$ (univariable)), while $\mathrm{N}, \mathrm{M}$ stage (TNM staging) and AJCC stage were only univariable significant (Table V lower), as it was easy to comprehend that metastasis and stage was related to patient outcome (21). These data indicated that risk score could directly predict patient survival.

\section{Discussion}

In the present study, we have identified and verified 46 survival related-biomarkers from 66 DEGs in CRC and then built a prognostic risk score which could be translated into the clinical setting. The 46 survival related-biomarkers mainly located in 

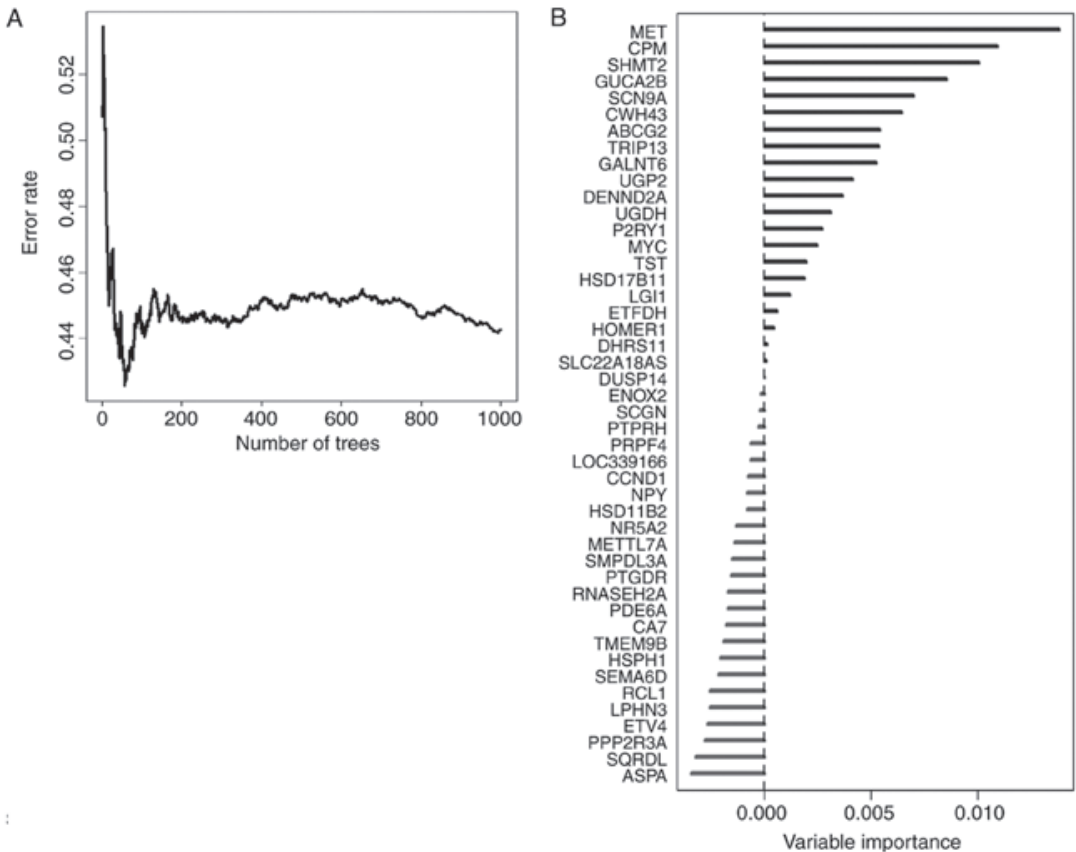

Figure 3. Survival related-DEGs ranked by variable importance. (A) Error rate of random survival forests algorithm (Ntree $=1,000$, default parameters of Hemant Ishwaran algorithm). (B) Variable importance of the 46 survival related-DEGs. DEGs, differentially expressed genes.

A

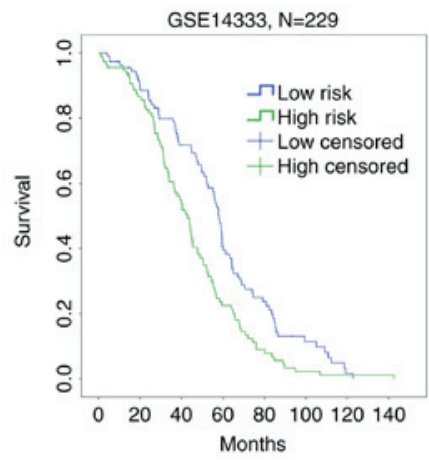

C

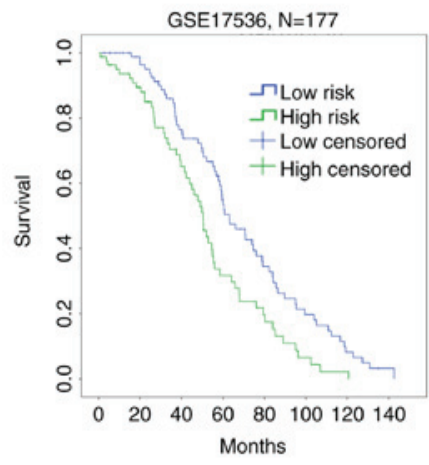

B

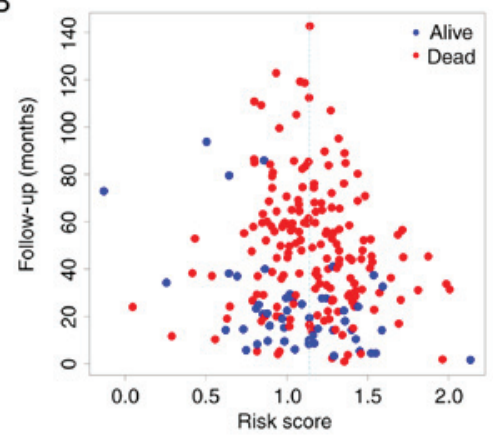

D

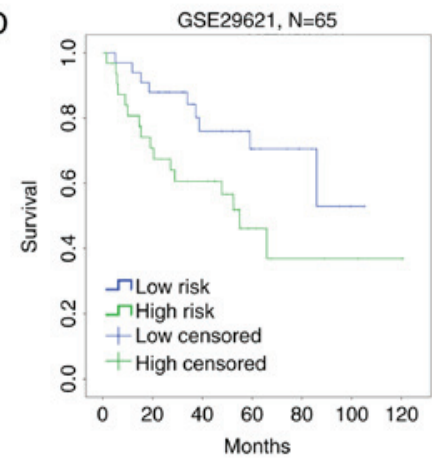

E

GSE14333
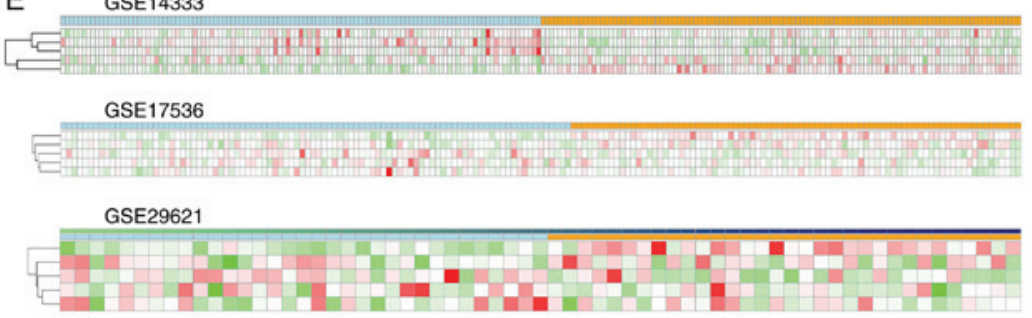

Figure 4. Test and validation of risk score in independent GEO datasets. (A) Kaplan-Meier survival curve of low and high risk patients in Training GSE14333 ( $\mathrm{P}=0.001$, by Mantel-Cox log rank). (B) Scatter diagram of live and dead outcome with different risk score value of GSE14333. Kaplan-Meier survival curve of low and high risk patients in validation set GSE17536 ( $P=0.001)$ (C) and GSE29621 ( $P=0.038$ ) (D). (E) Gene expression distribution of the 5 most important biomarkers in low and high risk patients in GSE14333, GSE17536 and GSE29621. Genes in GSE14333 were SCN9A, CPM, GUCA2B, MET and SHMT2 from top to bottom. Genes in GSE17536 and GSE29621 were SHMT2, MET, CPM, GUCA2B and SCN9A from top to bottom. GEO, Gene Expression Omnibus. 
Table V. Multivariable and univariable model tests of risk score and other factors.

A, GSE14333

\begin{tabular}{|c|c|c|c|c|c|c|c|c|}
\hline \multirow{3}{*}{$\begin{array}{l}\text { Variables } \\
\text { Risk score }\end{array}$} & \multicolumn{4}{|c|}{ Multivariable model } & \multicolumn{4}{|c|}{ Univariable model } \\
\hline & \multirow{2}{*}{$\begin{array}{c}\text { HR } \\
2.346\end{array}$} & \multicolumn{2}{|c|}{$95 \% \mathrm{CI}$ of $\mathrm{HR}$} & \multirow{2}{*}{$\begin{array}{c}\text { P-value } \\
0.005\end{array}$} & \multirow{2}{*}{$\begin{array}{c}\text { HR } \\
2.718\end{array}$} & \multicolumn{2}{|c|}{$95 \% \mathrm{CI}$ of $\mathrm{HR}$} & \multirow{2}{*}{$\begin{array}{l}\text { P-value } \\
0.001\end{array}$} \\
\hline & & 1.298 & 4.241 & & & 1.523 & 4.851 & \\
\hline Location & 0.965 & 0.814 & 1.144 & 0.683 & 0.892 & 0.76 & 1.047 & 0.163 \\
\hline Dukes stage & 1.18 & 0.926 & 1.503 & 0.18 & 1.044 & 0.86 & 1.266 & 0.666 \\
\hline Age of diagnosis & 1.008 & 0.994 & 1.023 & 0.257 & 1.105 & 1.002 & 1.028 & 0.02 \\
\hline Sex & 0.926 & 0.683 & 1.255 & 0.62 & 0.877 & 0.651 & 1.182 & 0.39 \\
\hline Adj XRT & 0.463 & 0.218 & 0.984 & 0.045 & 0.433 & 0.212 & 0.884 & 0.021 \\
\hline Adj CTX & 0.867 & 0.568 & 1.325 & 0.51 & 0.847 & 0.618 & 1.16 & 0.3 \\
\hline
\end{tabular}

\section{B, GSE17536}

Multivariable model

\begin{tabular}{|c|c|c|c|c|c|c|c|c|}
\hline \multirow{3}{*}{$\begin{array}{l}\text { Variables } \\
\text { Risk score }\end{array}$} & & & & & \\
\hline & \multirow{2}{*}{$\begin{array}{c}\mathrm{HR} \\
2.745\end{array}$} & \multicolumn{2}{|c|}{$95 \% \mathrm{CI}$ of $\mathrm{HR}$} & \multirow{2}{*}{$\begin{array}{c}\text { P-value } \\
0.016\end{array}$} & \multirow{2}{*}{$\begin{array}{c}\text { HR } \\
3.283\end{array}$} & \multicolumn{2}{|c|}{$95 \% \mathrm{CI}$ of $\mathrm{HR}$} & \multirow{2}{*}{$\begin{array}{c}\text { P-value } \\
0.003\end{array}$} \\
\hline & & 1.204 & 6.262 & & & 1.489 & 7.236 & \\
\hline Age & 1.015 & 0.999 & 1.031 & 0.061 & 1.018 & 1.003 & 1.034 & 0.016 \\
\hline Sex & 1.084 & 0.747 & 1.572 & 0.672 & 0.953 & 0.666 & 1.362 & 0.79 \\
\hline Ethnicity & 0.967 & 0.728 & 1.284 & 0.817 & 0.915 & 0.685 & 1.221 & 0.545 \\
\hline AJCC stage & 1.107 & 0.892 & 1.373 & 0.357 & 1.051 & 0.861 & 1.284 & 0.625 \\
\hline grade & 1.254 & 0.828 & 1.898 & 0.285 & 1.375 & 0.924 & 2.045 & 0.116 \\
\hline
\end{tabular}

C, GSE29621

Univariable model

\begin{tabular}{|c|c|c|c|c|c|c|c|c|}
\hline \multirow{3}{*}{$\frac{\text { Variables }}{\text { Risk score }}$} & \multicolumn{4}{|c|}{ Multivariable model } & \multicolumn{4}{|c|}{ Univariable model } \\
\hline & \multirow{2}{*}{$\begin{array}{c}\text { HR } \\
9.03\end{array}$} & \multicolumn{2}{|c|}{$95 \% \mathrm{CI}$ of $\mathrm{HR}$} & \multirow{2}{*}{$\begin{array}{c}\text { P-value } \\
0.019\end{array}$} & \multirow{2}{*}{$\begin{array}{c}\mathrm{HR} \\
2.526\end{array}$} & \multicolumn{2}{|c|}{$95 \% \mathrm{CI}$ of $\mathrm{HR}$} & \multirow{2}{*}{$\begin{array}{c}\text { P-value } \\
2.73 \mathrm{E}-05\end{array}$} \\
\hline & & 1.425 & 57.223 & & & 0.481 & 13.269 & \\
\hline Sex & 1.243 & 0.513 & 3.014 & 0.63 & 1.508 & 0.649 & 3.505 & 0.34 \\
\hline T stage & 0.449 & 0.091 & 2.209 & 0.325 & 1.048 & 0.438 & 2.509 & 0.915 \\
\hline $\mathrm{N}$ stage & 1.583 & 0.604 & 4.143 & 0.35 & 2.688 & 1.526 & 4.734 & 0.001 \\
\hline M stage & 2.065 & 0.368 & 11.592 & 0.41 & 4.934 & 2.188 & 11.124 & $1.19 \mathrm{E}-04$ \\
\hline Histology grade & 0.849 & 0.325 & 2.219 & 0.738 & 0.665 & 0.284 & 1.558 & 0.348 \\
\hline AJCC stage & 1.965 & 0.518 & 7.45 & 0.321 & 2.708 & 1.615 & 4.542 & $1.59 \mathrm{E}-04$ \\
\hline
\end{tabular}

HR, hazard ratio; Adj XRT, adjuvant radiation therapy; Adj CTX, adjuvant chemotherapy.

cytoplasm, membrane and nucleolus, only a small portion in mitochondria and other sub-cellular parts. Their GO enrichment showed that these genes involved in multiple biological processes such as response to drug, metabolic process, cell proliferation, and positive regulation of cell proliferation. Obviously, these biological processes played a pivotal role in cancer proliferation, drug-resistance, and metastasis, thus further affecting patient survival (22-24). Genes like MYC and CCND1 within CRC pathway in KEGG annotation also participated in other cancer pathway as endometrial cancer or chronic myeloid leukemia $(25,26)$. After that, we ranked the
46 survival-related genes by random survival forests algorithm and got five most important biomarkers namely MET, CPM, SHMT2, GUCA2B and SCN9A.

Recently, MET was reported gradually upregulated in the development and progression of CRC from normal epithelium to adenoma, colorectal carcinoma and metastases $(27,28)$. Although others argued that the increase of MET in metastatic CRC was an acquired response to EGFR inhibition, not a de novo phenomenon (29), its prognostic value was confirmed by several independent researches $(30,31)$. Moreover, suppressing MET by specific inhibitor or shRNA has a therapeutic role in 
CRC $(32,33)$. CPM was less reported, and only one literature revealed that it was the target of miR-146a which promoted cell migration and invasion in CRC via CPM/src-FAK pathway (34). It was suggested that CPM has the potential to be a therapeutic target in cancer (35), but its function still need further discovery. SHMT2 participated in the cellular one-carbon metabolism, and has been implicated as a critical component for tumor survival. Its upregulation was correlated with tumor proliferation in several cancers $(36,37)$. Kim et al found SHMT2 activity limits that of pyruvate kinase (PKM2) and reduces oxygen consumption, thus eliciting a metabolic switch that confers a profound survival advantage to cells in poorly vascularized regions (38). GUCA2B and SCN9A were rarely demonstrated in cancer and more light should shed on their role in CRC. The cause and progression of CRC are complicated and remains to be further elucidated, and we think the rest genes in Table III should have potential value in better interpreting the carcinogenesis and progression of CRC.

Moreover, we established a linear risk score as a survival predicting model based on the above five genes by multivariable Cox regression using highly reliable CRC datasets. This risk score predicted patients at high risk of mortality independently and directly in all validation datasets. Although more prospective studies are necessary to further validate the reliability and robustness of this risk score, our work provide an new method toward clinical applications of gene expression profiling in $\mathrm{CRC}$, especially in future personalized prediction and precision medicine.

\section{Acknowledgements}

The present study was supported by grants from the Ministry of Science and Technology of China (grant no. 2013CB945401) and the National Natural Science Foundation of China (grant no. 81500503 ). Data which were not shown in this article could be archived by contacting the corresponding author.

\section{References}

1. Torre LA, Bray F, Siegel RL, Ferlay J, Lortet-Tieulent J and Jemal A: Global cancer statistics, 2012. CA Cancer J Clin 65: 87-108, 2015

2. Siegel RL, Miller KD and Jemal A: Cancer Statistics, 2017. CA Cancer J Clin 67: 7-30, 2017.

3. Frampton $M$ and Houlston RS: Modeling the prevention of colorectal cancer from the combined impact of host and behavioral risk factors. Genet Med 19: 314-321, 2017.

4. Inadomi JM: Screening for colorectal neoplasia. N Engl J Med 376: 149-156, 2017.

5. Chang W, Gao X, Han Y, Du Y, Liu Q, Wang L, Tan X, Zhang Q, Liu Y, Zhu Y, et al: Gene expression profiling-derived immunohistochemistry signature with high prognostic value in colorectal carcinoma. Gut 63: 1457-1467, 2014.

6. Shahid M, Choi TG, Nguyen MN, Matondo A, Jo YH, Yoo JY, Nguyen NN, Yun HR, Kim J, Akter S, et al: An 8-gene signature for prediction of prognosis and chemoresponse in non-small cell lung cancer. Oncotarget 7: 86561-86572, 2016.

7. Francois-Vaughan H, Adebayo AO, Brilliant KE, Parry NMA, Gruppuso PA and Sanders JA: Persistent effect of mTOR inhibition on preneoplastic foci progression and gene expression in a rat model of hepatocellular carcinoma. Carcinogenesis 37: 408-419, 2016

8. Baek SJ, Sato K, Nishida N, Koseki J, Azuma R, Kawamoto K, Konno M, Hayashi K, Satoh T, Doki Y, et al: MicroRNA miR-374, a potential radiosensitizer for carbon ion beam radiotherapy. Oncol Rep 36: 2946-2950, 2016.
9. Paula LM, De Moraes LH, Do Canto AL, Dos Santos L, Martin AA, Rogatto SR and De Azevedo Canevari R: Analysis of molecular markers as predictive factors of lymph node involvement in breast carcinoma. Oncol Lett 13: 488-496, 2017.

10. Li G, Li X, Yang M, Xu L, Deng S and Ran L: Prediction of biomarkers of oral squamous cell carcinoma using microarray technology. Sci Rep 7: 42105, 2017.

11. Viziteu E, Klein B, Basbous J, Lin YL, Hirtz C, Gourzones C, Tiers L, Bruyer A, Vincent L, Grandmougin C, et al: RECQ1 helicase is involved in replication stress survival and drug resistance in multiple myeloma. Leukemia: Mar 10, 2017 (Epub ahead of print).

12. Wu J, Wang J and Shen W: Identification of MAGEA12 as a prognostic outlier gene in gastric cancers. Neoplasma 64: 238-243, 2017.

13. Jo J, Nam CM, Sull JW, Yun JE, Kim SY, Lee SJ, Kim YN, Park EJ, Kimm H and Jee SH: Prediction of colorectal cancer risk using a genetic risk score: The Korean cancer prevention study-II (KCPS-II). Genomics Inform 10: 175-183, 2012.

14. Ito H, Mo Q, Qin LX, Viale A, Maithel SK, Maker AV, Shia J, Kingham P, Allen P, DeMatteo RP, et al: Gene expression profiles accurately predict outcome following liver resection in patients with metastatic colorectal cancer. PLoS One 8: e81680, 2013.

15. Mármol I, Sánchez-de-Diego C, Pradilla Dieste A, Cerrada E and Rodriguez Yoldi MJ: Colorectal carcinoma: A general overview and future perspectives in colorectal cancer. Int J Mol Sci 18: pii:E197, 2017.

16. Zhu Q, Izumchenko E, Aliper AM, Makarev E, Paz K, Buzdin AA, Zhavoronkov AA and Sidransky D: Pathway activation strength is a novel independent prognostic biomarker for cetuximab sensitivity in colorectal cancer patients. Hum Genome Var 2: 15009, 2015.

17. Zhu T, Gao YF, Chen YX, Wang ZB, Yin JY, Mao XY, Li X, Zhang W, Zhou HH and Liu ZQ: Genome-scale analysis identifies GJB2 and ERO1LB as prognosis markers in patients with pancreatic cancer. Oncotarget 8: 21281-21289, 2017.

18. Tabas-Madrid D, Nogales-Cadenas R and Pascual-Montano A: GeneCodis3: A non-redundant and modular enrichment analysis tool for functional genomics. Nucleic Acids Res 40: W478-W483, 2012.

19. Gruber-Rouh T, Marko C, Thalhammer A, Nour-Eldin NE, Langenbach M, Beeres M, Naguib NN, Zangos S and Vogl TJ: Current strategies in interventional oncology of colorectal liver metastases. Br J Radiol: May 26, 2016 (Epub ahead of print).

20. Song N, Shin A, Park JW, Kim J and Oh JH: Common risk variants for colorectal cancer: An evaluation of associations with age at cancer onset. Sci Rep 7: 40644, 2017.

21. Slattery ML, Herrick JS, Mullany LE, Gertz J and Wolff RK: Improved survival among colon cancer patients with increased differentially expressed pathways. BMC Med 13: 75, 2015.

22. Dong G, Mao Q, Yu D, Zhang Y, Qiu M, Dong G, Chen Q, Xia W, Wang J, Xu L and Jiang F: Integrative analysis of copy number and transcriptional expression profiles in esophageal cancer to identify a novel driver gene for therapy. Sci Rep 7: 42060, 2017.

23. Gao B, Shao Q, Choudhry H, Marcus V, Dong K, Ragoussis J and Gao ZH: Weighted gene co-expression network analysis of colorectal cancer liver metastasis genome sequencing data and screening of anti-metastasis drugs. Int J Oncol 49: 1108-1118, 2016.

24. Xiong W, Gao D, Li Y, Liu X, Dai P, Qin J, Wang G, Li K, Bai H and $\mathrm{Li} \mathrm{W}$ : Genome-wide profiling of chemoradiation-induced changes in alternative splicing in colon cancer cells. Oncol Rep 36: 2142-2150, 2016.

25. Huang H, Jiang X, Wang J, Li Y, Song CX, Chen P, Li S, Gurbuxani S, Arnovitz S, Wang Y, et al: Identification of MLL-fusion/MYC-miR-26-TET1 signaling circuit in MLL-rearranged leukemia. Cancer Lett 372: 157-165, 2016.

26. Zhu XL, Ai ZH, Wang J, Xu YL and Teng YC: Weighted gene co-expression network analysis in identification of endometrial cancer prognosis markers. Asian Pac J Cancer Prev 13: 4607-4611, 2012

27. Bradley CA, Dunne PD, Bingham V, McQuaid S, Khawaja H, Craig S, James J, Moore WL, McArt DG, Lawler M, et al: Transcriptional upregulation of c-MET is associated with invasion and tumor budding in colorectal cancer. Oncotarget 7: 78932-78945, 2016.

28. Gayyed MF, Abd El-Maqsoud NM, El-Hameed El-Heeny AA and Mohammed MF: c-MET expression in colorectal adenomas and primary carcinomas with its corresponding metastases. J Gastrointest Oncol 6: 618-627, 2015. 
29. Raghav K, Morris V, Tang C, Morelli P, Amin HM, Chen K, Manyam GC, Broom B, Overman MJ, Shaw K, et al: MET amplification in metastatic colorectal cancer: An acquired response to EGFR inhibition, not a de novo phenomenon. Oncotarget 7 : 54627-54631, 2016.

30. Al-Maghrabi J, Emam E, Gomaa W, Saggaf M, Buhmeida A, Al-Qahtani M and Al-Ahwal M: c-MET immunostaining in colorectal carcinoma is associated with local disease recurrence. BMC Cancer 15: 676, 2015.

31. Takahashi N, Iwasa S, Taniguchi H, Sasaki Y, Shoji H, Honma Y, Takashima A, Okita N, Kato K, Hamaguchi T, et al: Prognostic role of ERBB2, MET and VEGFA expression in metastatic colorectal cancer patients treated with anti-EGFR antibodies. $\mathrm{Br}$ J Cancer 114: 1003-1011, 2016.

32. Jia Y, Dai G, Wang J, Gao X, Zhao Z, Duan Z, Gu B, Yang W, $\mathrm{Wu} \mathrm{J}, \mathrm{Ju} Y$, et al: c-MET inhibition enhances the response of the colorectal cancer cells to irradiation in vitro and in vivo. Oncol Lett 11: 2879-2885, 2016.

33. Sun Y, Sun L, An Y and Shen X: Cabozantinib, a Novel c-Met Inhibitor, inhibits colorectal cancer development in a Xenograft model. Med Sci Monit 21: 2316-2321, 2015.

34. Lu D, Yao Q, Zhan C, Le-Meng Z, Liu H, Cai Y, Tu C, Li X, Zou Y and Zhang S: MicroRNA-146a promote cell migration and invasion in human colorectal cancer via carboxypeptidase M/src-FAK pathway. Oncotarget 8: 22674-22684, 2017.
35. Denis CJ and Lambeir AM: The potential of carboxypeptidase $\mathbf{M}$ as a therapeutic target in cancer. Expert Opin Ther Targets 17: 265-279, 2013.

36. Wang B, Wang W, Zhu Z, Zhang X, Tang F, Wang D, Liu X, Yan X and Zhuang H: Mitochondrial serine hydroxymethyltransferase 2 is a potential diagnostic and prognostic biomarker for human glioma. Clin Neurol Neurosurg 154: 28-33, 2017.

37. Zhang L, Chen Z, Xue D, Zhang Q, Liu X, Luh F, Hong L, Zhang H, Pan F, Liu Y, et al: Prognostic and therapeutic value of mitochondrial serine hydroxyl-methyltransferase 2 as a breast cancer biomarker. Oncol Rep 36: 2489-2500, 2016.

38. Kim D, Fiske BP, Birsoy K, Freinkman E, Kami K, Possemato RL, Chudnovsky Y, Pacold ME, Chen WW, Cantor JR, et al: SHMT2 drives glioma cell survival in ischaemia but imposes a dependence on glycine clearance. Nature 520: 363-367, 2015. 